\title{
On the complete model with stochastic volatility by Hobson and Rogers
}

\author{
By Marco Di Francesco and Andrea PASCuCCI \\ Dipartimento di Matematica, Università di Bologna, Piazza di Porta S. Donato 5, \\ 40126 Bologna, Italy (difrance@dm.unibo.it; pascucci@dm.unibo.it)
}

Received 6 August 2003; accepted 16 June 2004

In the complete model with stochastic volatility by Hobson and Rogers, preference independent options prices are solutions to degenerate partial differential equations obtained by including additional state variables describing the dependence on past prices of the underlying. In this paper, we aim to emphasize the mathematical tractability of the model by presenting analytical and numerical results comparable with the known ones in the classical Black-Scholes environment.

Keywords: Black-Scholes model; stochastic volatility; path-dependent contingent claim; Kolmogorov equation; hypoelliptic equation

\section{Introduction}

It is widely believed that the celebrated Black-Scholes model (Black \& Scholes 1973) cannot describe option prices dynamics in real markets. The characteristic observed effects of 'smiles' and 'skews' contrast with the assumption of constant volatility of the underlying asset. Indeed there is strong evidence that volatility depends in some way on the present stock price (leverage effect) and on realization of past prices (volatility persistence), as well as on the moneyness, time and maturity (volatility term structure): we refer, for instance, to Tompkins (2001), Derman \& Kani (1998), Ghysels et al. (1996) and Bollerslev et al. (1992).

For this reason many authors have developed variations of the Black-Scholes model, aiming to capture at least some of the above observed features. Within the framework of continuous Itô processes, these alternative theories divide into two broad classes: single-factor models, in which the volatility is a deterministic function of present or past values of the underlying price, and multi-factor models, which allow for exogenous stochastic influence (see, for instance, the monograph by Epps (2000) and the recent papers by Barucci et al. (2003) and Davis (2004) for comprehensive bibliographic references).

An apparent advantage of single- over multi-factor models is that the Brownian motion which drives the asset price is the only source of randomness. Then the arbitrage argument that underlies the Black-Scholes model is preserved and there are unique preference-independent prices for contingent claims. The simplest singlefactor model is the so-called level-dependent volatility in which the underlying price 
$S_{t}$ satisfies

$$
\mathrm{d} S_{t}=\mu S_{t} \mathrm{~d} t+\sigma S_{t} \mathrm{~d} B_{t},
$$

where $B$ is a Brownian motion and $\sigma=\sigma\left(t, S_{t}\right)$ is a deterministic function of the current value $S_{t}$ and time. Special cases are the CEV model by Cox \& Ross (1976), where $\left.\sigma\left(S_{t}\right)=S_{t}^{\alpha-1}, \alpha \in\right] 0,1[$, and the implied tree models by Derman \& Kani (1998), Dupire (1997) and Rubinstein (1994).

Empirical analysis has shown that level-dependent volatility still fails to price derivative securities better than the usual Black-Scholes model does. This is the conclusion of the study by Dumas et al. (1998), who suggest a volatility related to past changes of the underlying prices, as the last candidate model before resorting to fully stochastic volatility.

An interesting class of such models was proposed by Hobson \& Rogers. In Hobson \& Rogers (1998) a specific version is examined in which the volatility is defined in terms of the difference between the current price and an exponential average of past prices. As in the discrete-time ARCH and GARCH environments, this is designed to reflect the perception that large movements of the asset price in the past tend to forecast higher future volatility. In Hobson \& Rogers (1998) it is shown that the model indeed exhibits a wide variety of smiles and skews, and can account for volatility term structures as the average of prices evolves through time (see also the study by Figà-Talamanca \& Guerra (2000)). Hobson \& Rogers claim to solve numerically the associated partial differential equation (PDE) by a finite-difference method: unfortunately no scheme is explicitly given in Hobson \& Rogers (1998), nor are convergence results proved. Actually, it is difficult to obtain these results by the 'classical' theory of parabolic PDEs. Indeed, incorporating past prices enters an additional state variable on which the derivative's arbitrage-free price depends. Then, as in the case of path-dependent contingent claims such as Asian or look-back options, the augmented PDE associated to the model is strongly degenerate and standard analytical and numerical techniques for uniformly parabolic equations do not apply.

On the other hand, we recall that Barucci et al. (2001) successfully studied the problem of pricing Asian options in the framework of PDEs of Kolmogorov type. This class of degenerate diffusion PDEs, whose precise definition is given in $\S 2$, shares most of the prominent features of classical parabolic equations.

In this paper we present analytical and numerical results on Kolmogorov PDEs with variable coefficients which apply to the Hobson-Rogers model for European and Asian-style derivatives. Consequently, we wish to emphasize the mathematical tractability of this model. Indeed our results are comparable with those known for the classical Black-Scholes environment. In particular, we aim to propose a finitedifference scheme for the Hobson-Rogers model and prove convergence results.

Let us recall that nonlinear Kolmogorov equations have also been recently considered by Peszek (1995) for pricing options with memory feedback and by Antonelli \& Pascucci (2002) and Pascucci \& Polidoro (2003) for a stochastic differential utility problem.

The paper is organized as follows. In the next section, we present theoretical results about Kolmogorov PDEs which allow us to treat a variety of path-dependent contingent claims. In $\S 3$, we prove numerical results by suitably adapting finitedifferences schemes to the degenerate setting.

Proc. R. Soc. Lond. A (2004) 


\section{Kolmogorov equations}

Degenerate PDEs of Kolmogorov type naturally arise in the problem of pricing pathdependent contingent claims. The simplest significant example is given by Asian-style derivatives: if we assume that the stock price $S_{t}$ is a lognormal stochastic process, i.e. a solution to the stochastic differential equation (1.1) with $\mu$ and $\sigma$ being fixed constants, then the price $U$ of a geometric average Asian option is a solution to the equation

$$
\partial_{t} U+r S \partial_{S} U+\frac{1}{2} \sigma^{2} S^{2} \partial_{S S} U+\log (S) \partial_{A} U=r U,
$$

where ' $A$ ' denotes the path-dependent variable and $r$ is the risk-free rate (see, for example, Wilmott et al. 1993). By an elementary change of variables, (2.1) can be reduced to the following $\mathrm{PDE}$ in $\mathbb{R}^{3}$ :

$$
\partial_{x_{1} x_{1}} u+x_{1} \partial_{x_{2}} u-\partial_{t} u=0 .
$$

Although (2.2) is strongly degenerate, in the sense that there is a complete lack of diffusion in the $x_{2}$-direction, Kolmogorov (1934) constructed an explicit fundamental solution to (2.2) of Gaussian type, which is a $C^{\infty}$ function outside the diagonal (cf. (2.12)). Consequently, equation (2.2) has a closed-form solution and is hypoelliptic, that is, every distributional solution to $(2.2)$ is a $C^{\infty}$ function. As we shall see later, the Kolmogorov method can be applied in a very general framework.

In the Hobson-Rogers model (Hobson \& Rogers 1998), the stock price $S_{t}$ is described by an Itô process of the form

$$
\mathrm{d} S_{t}=\mu\left(D_{t}\right) S_{t} \mathrm{~d} t+\sigma\left(D_{t}\right) S_{t} \mathrm{~d} B_{t},
$$

where $D_{t}$ represents the deviation from the trend and is defined as the difference between the current value and a geometric, exponentially weighted average of past prices. More precisely, if $Z_{t}=\log \left(\mathrm{e}^{-r t} S_{t}\right)$ denotes the discounted log-price, then

$$
D_{t}=Z_{t}-\int_{0}^{+\infty} \lambda \mathrm{e}^{-\lambda \tau} Z_{\tau}-\tau \mathrm{d} \tau
$$

where $\lambda$ is a positive parameter. Hobson \& Rogers prove the existence of an equivalent probability measure under which the discounted price $\mathrm{e}^{-r t} S_{t}$ is a martingale. Then usual no-arbitrage arguments can be used to determine the price of the derivative.

In Hobson \& Rogers (1998), it is proved that the price $U_{t}$ of a European-style contingent claim with terminal pay-off $U_{T}=\varphi\left(S_{T}\right)$ is given by

$$
U_{t}=u\left(\log \left(S_{t}\right), \log \left(S_{t}\right)-D_{t}, T-t\right),
$$

where $u=u\left(x_{1}, x_{2}, t\right)$ is the solution to the following degenerate PDE with variable coefficients in $\mathbb{R}^{3}$ :

$$
\frac{1}{2} \sigma^{2}\left(x_{1}-x_{2}\right)\left(\partial_{x_{1} x_{1}} u-\partial_{x_{1}} u\right)+\lambda\left(x_{1}-x_{2}\right) \partial_{x_{2}} u-\partial_{t} u=0,
$$

with initial datum $u\left(x_{1}, x_{2}, 0\right)=\varphi\left(\mathrm{e}^{x_{1}}\right)$.

The same argument can be used for the more general case of a geometric average Asian option. Let $A_{t}$ be the path-dependent process describing the geometric average of the underlying:

$$
\mathrm{d} A_{t}=\log \left(S_{t}\right) \mathrm{d} t .
$$

Proc. R. Soc. Lond. A (2004) 
The price $U_{t}$ of a derivative with terminal pay-off $U_{T}=\varphi\left(S_{T}, A_{T}\right)$ is then given by

$$
U_{t}=u\left(\log \left(S_{t}\right), \log \left(S_{t}\right)-D_{t}, A_{t}, T-t\right),
$$

where $u=u\left(x_{1}, x_{2}, x_{3}, t\right)$ is the solution to the PDE

$$
\frac{1}{2} \sigma^{2}\left(x_{1}-x_{2}\right)\left(\partial_{x_{1} x_{1}} u-\partial_{x_{1}} u\right)+\lambda\left(x_{1}-x_{2}\right) \partial_{x_{2}} u+x_{1} \partial_{x_{3}} u-\partial_{t} u=0
$$

with initial datum $u\left(x_{1}, x_{2}, x_{3}, 0\right)=\varphi\left(\mathrm{e}^{x_{1}}, x_{3}\right)$. We emphasize that $(2.5)$ is a PDE in $\mathbb{R}^{4}$ with diffusion only in the $x_{1}$-direction.

The above examples motivate the study of the following class of degenerate PDEs.

Definition 2.1. We call the Kolmogorov equation (in short, KE) a PDE of the form

$$
K u \equiv \sum_{i, j=1}^{p}\left(a_{i j}(x, t) \partial_{x_{i} x_{j}} u+c_{i}(x, t) \partial_{x_{i}} u\right)+\sum_{i, j=1}^{N} b_{i j} x_{i} \partial_{x_{j}} u-\partial_{t} u=0,
$$

where $\left(x_{1}, \ldots, x_{N}, t\right) \equiv(x, t) \equiv z \in \mathbb{R}^{N+1}$, under the following structural conditions:

(i) there exists $\mu>0$ such that

$$
\mu^{-1}|\xi|^{2}<\sum_{i, j=1}^{p} a_{i j}(z) \xi_{i} \xi_{j}<\mu|\xi|^{2}
$$

for every $\xi \in \mathbb{R}^{p}$ and $z \in \mathbb{R}^{N+1}$;

(ii) the matrix $B=\left(b_{i j}\right)$ is constant and has the following block form:

$$
\left(\begin{array}{ccccc}
* & B_{1} & 0 & \cdots & 0 \\
* & * & B_{2} & \cdots & 0 \\
\vdots & \vdots & \vdots & \ddots & \vdots \\
* & * & * & \cdots & B_{m} \\
* & * & * & \cdots & *
\end{array}\right)
$$

where the $B_{j}$-valued blocks are $p_{j-1} \times p_{j}$ matrices of rank $p_{j}, j=1,2, \ldots, m$, and the blocks denoted by ' $*$ ' are arbitrary. Moreover, the $p_{j}$ values are positive integers such that

$$
p=p_{0} \geqslant p_{1} \geqslant \cdots \geqslant p_{m} \geqslant 1, \quad \text { and } \quad p+p_{1}+\cdots+p_{m}=N .
$$

Example 2.2. Equation (2.2) is obviously a KE; in this case

$$
B=\left(\begin{array}{ll}
0 & 1 \\
0 & 0
\end{array}\right) \text {. }
$$

It is also clear that every parabolic equation is a $\mathrm{KE}$.

To verify that equations (2.4) and (2.5) of the Hobson-Rogers model are KEs, it is convenient to recall that the structural assumptions of definition 2.1 are equivalent to the following classical Hörmander condition (Hörmander 1967) (see, for instance, Lanconelli \& Polidoro 1994). The well-known Hörmander condition gives a characterization of hypoelliptic degenerate PDEs with smooth coefficients. In our setting it reads as follows. 
Condition 2.3 (Hörmander condition). The first-order differential operators

$$
\partial_{x_{1}}, \ldots, \partial_{x_{p}} \text { and } Y=\sum_{i, j=1}^{N} b_{i j} x_{i} \partial_{x_{j}}-\partial_{t},
$$

together with their commutators, span $\mathbb{R}^{N+1}$ at every point.

Example 2.4. Equation (2.4) is a KE. Indeed, for every $\lambda>0$, the first-order differential operators

$$
X \equiv \partial_{x_{1}}, \quad Y=\lambda\left(x_{1}-x_{2}\right) \partial_{x_{2}}-\partial_{t}, \quad[X, Y] \equiv X Y-Y X=\lambda \partial_{x_{2}}
$$

are linearly independent at every point and form a basis of $\mathbb{R}^{3}$. Analogously, equation (2.5) is a KE since

$$
\begin{gathered}
X=\partial_{x_{1}}, \quad \tilde{Y}=\lambda\left(x_{1}-x_{2}\right) \partial_{x_{2}}+x_{1} \partial_{x_{3}}-\partial_{t} \\
{[X, \tilde{Y}]=\lambda \partial_{x_{2}}+\partial_{x_{3}}, \quad[\tilde{Y},[X, \tilde{Y}]]=\lambda^{2} \partial_{x_{2}}}
\end{gathered}
$$

span $\mathbb{R}^{4}$ at every point.

Definition 2.5. A KE in the form (2.6) with constant $a_{i j}$ and $c_{i} \equiv 0$ for $i, j=$ $1, \ldots, p$ is called a 'Kolmogorov equation with constant coefficients'.

The denomination 'KE with constant coefficients' stems from the theory of parabolic PDEs. Indeed, a constant-coefficients parabolic equation is nothing more than a translation-invariant equation on the Euclidean space. Similarly, a constantcoefficients KE in the form (2.6) has the remarkable property of being invariant with respect to the non-Euclidean left translations in the Lie group law

$$
(x, t) \circ(y, s)=(y+E(s) x, t+s), \quad(x, t),(y, s) \in \mathbb{R}^{N+1},
$$

where $E(t)=\exp \left(-t B^{\mathrm{T}}\right)$, and $B^{\mathrm{T}}$ denotes the transpose of the matrix $B$.

In this case, the explicit expression of $\Gamma(z ; \zeta)$, the fundamental solution of $(2.6)$ evaluated in $z$ with a pole at $\zeta$, is given by $\Gamma(z ; \zeta)=\Gamma\left(\zeta^{-1} \circ z ; 0\right)$, where

$$
\Gamma(x, t ; 0,0)=\frac{(4 \pi)^{-N / 2}}{\sqrt{\operatorname{det} C(t)}} \exp \left(-\frac{1}{4}\left\langle C^{-1}(t) x, x\right\rangle-t \operatorname{tr}(B)\right),
$$

for $(x, t) \in \mathbb{R}^{N} \times \mathbb{R}^{+}$and $C(t)$ is the positive definite matrix

$$
C(t)=\int_{0}^{t} E(s) A E^{\mathrm{T}}(s) \mathrm{d} s, \quad t>0 .
$$

As usual, a solution to the Cauchy problem for (2.6) can be expressed in closed form, as the convolution of the initial datum with the fundamental solution $\Gamma$. As in classical theory, constant-coefficients KEs serve as an essential class of prototypes and many results can be extended to the general situation of variable coefficients by perturbation arguments (see, for example, the survey by Lanconelli et al. (2002)).

Among constant-coefficient KEs, a particularly important role is played by equations for which all the $*$-blocks in (2.8) are zero matrices. Indeed these KEs have the remarkable property of being homogeneous with respect to the dilations $\left(\delta_{\lambda}\right)_{\lambda>0}$ in $\mathbb{R}^{N+1}$ defined by

$$
\delta_{\lambda}=\operatorname{diag}\left(\lambda I_{p_{0}}, \lambda^{3} I_{p_{1}}, \ldots, \lambda^{2 m+1} I_{p_{m}}, \lambda^{2}\right),
$$


where $I_{p_{j}}$ denotes the $p_{j} \times p_{j}$ identity matrix. More precisely, it holds that

$$
K \circ \delta_{\lambda}=\lambda^{2}\left(\delta_{\lambda} \circ K\right), \quad \forall \lambda>0 .
$$

Subelliptic operators on homogeneous Lie groups were first studied by Folland (1975).

Note that equation (2.2) is homogeneous with respect to the dilations

$$
\delta_{\lambda}\left(x_{1}, x_{2}, t\right)=\left(\lambda x_{1}, \lambda^{3} x_{2}, \lambda^{2} t\right) .
$$

In contrast, in the KEs arising in the Hobson-Rogers model, not all the $*$-blocks are null.

The main result of this section is the following theorem, proved in Di Francesco \& Pascucci (2004) (see also Polidoro (1994) for the case of null $*$-blocks). In the next statement, $\bar{\partial}_{x}=\left(\partial_{x_{0}}, \ldots, \partial_{x_{p}}\right)$ denotes the gradient in the first $p$ variables, $Y_{z}$ and $Y_{\zeta}$, respectively, denote the first-order differential operators in (2.9) acting on the variables $z=(x, t)$ and $\zeta=(y, s)$. Moreover, we say that a function $\varphi$ is slowly increasing if

$$
|\varphi(x)| \leqslant \alpha \mathrm{e}^{\beta|x|^{2}}, \quad \forall x \in \mathbb{R}^{N},
$$

for some positive constants $\alpha, \beta$.

Theorem 2.6. Assume that the coefficients of the KE (2.6) are infinitely differentiable in $\left.\mathbb{R}^{N} \times\right] 0, T$ [ and that all the derivatives are bounded functions. Then (2.6) has a fundamental solution $\Gamma$ : in particular, for every $\varphi \in C\left(\mathbb{R}^{N}\right)$, slowly increasing with suitably small $\beta$ in (2.16), the function

$$
u(x, t)=\int_{\mathbb{R}^{N}} \Gamma(x, t ; y, 0) \varphi(y) \mathrm{d} y
$$

is the unique slowly increasing, classical solution to the Cauchy problem

$$
\left.\begin{array}{rlrl}
K u=0 & & \text { in } \left.\mathbb{R}^{N} \times\right] 0, T[, \\
u(\cdot, 0)=\varphi & & \text { in } \mathbb{R}^{N} .
\end{array}\right\}
$$

Moreover, the function $\Gamma(z ; \zeta)$ is infinitely differentiable for $z \neq \zeta$ and we have the following estimate of the derivatives:

$$
\left|\bar{\partial}_{x}^{h} \bar{\partial}_{y}^{k} Y_{z}^{m} Y_{\zeta}^{n} \Gamma(x, t ; y, s)\right| \leqslant c(t-s)^{(-(|h|+|k|) / 2)-m-n} \Gamma_{\mu}(x, t ; y, s),
$$

where $\Gamma_{\mu}$ is the fundamental solution of the constant-coefficients $K E$ with $\left(a_{i j}\right)=\mu I_{p}$ and $\mu$ as in (2.7). The explicit expression of $\Gamma_{\mu}$ is given in (2.12). The constant $c$ depends on the multi-indexes $h, k$ and on the non-negative integers $m, n$.

Remark 2.7. The hypotheses on the regularity of the coefficients in the previous statement can be considerably relaxed. For the existence of $\Gamma$, it is sufficient to assume that the coefficients are bounded and Hölder continuous. Analogously, estimate (2.19) still holds true under the usual hypotheses: the regularity of order $m+\alpha, \alpha>0$, of the coefficients implies an estimate of the derivatives of order $m+2$ of $\Gamma$. Note that estimate (2.19) involves only the first $p$ spatial derivatives $\partial_{x_{1}}, \ldots, \partial_{x_{p}}$ and the vector field $Y$. If we consider $\bar{\partial}_{x}$ as first-order derivatives, then $Y$ weighs as a second-order derivative, in perfect analogy with the classical parabolic case. Estimates for the other spatial derivatives can be obtained from (2.19) by the Hörmander condition: for instance, in the case of equation (2.4), by (2.10) we have $\partial_{x_{2}}=\partial_{x_{1}} Y-Y \partial_{x_{1}}$ so that $\partial_{x_{2}}$ should be considered as a third-order derivative. 


\section{A numerical scheme}

We are concerned with the numerical solution to the Cauchy problem for the KE (2.4). Here we extend some results by Barucci et al. (2001) for the homogeneous KE (2.2) with constant coefficients. As we shall see below, the main new difficulties in treating the Hobson-Rogers equation (2.4) are due to the lack of homogeneity. Our scheme allows us to consider the problem

$$
\begin{aligned}
a \partial_{x x} u+b \partial_{x} u+(\alpha x+\beta y) \partial_{y} u-\partial_{t} u=0 & & \text { in } \left.S_{T} \equiv \mathbb{R}^{2} \times\right] 0, T[, \\
u(\cdot, 0)=\varphi & & \text { in } \mathbb{R}^{2},
\end{aligned}
$$

where $a=a(x, y, t) \geqslant a_{0}>0$ and $\alpha, \beta \in \mathbb{R}, \alpha \neq 0$. Preferring straightforwardness to generality, we assume that the coefficients $a, b$ are smooth functions with bounded derivatives. We remark explicitly that the condition $\alpha \neq 0$ ensures that (3.1) is a $\mathrm{KE}$, since it verifies the Hörmander condition.

We first consider the case of the model equation

$$
K u \equiv \partial_{x x} u+(x-y) \partial_{y} u-\partial_{t} u=0,
$$

corresponding to $a=\alpha=1=-\beta$ and $b=0$ in (3.1). In view of the theory of KEs, it seems natural to consider and approximate the first-order operators related to the equation as in (2.9), rather than the Euclidean derivatives as usual. Thus, as in example 2.4, we consider, for fixed $x, y$, the first-order part of $K$ as a unique 'directional' derivative, which we denote by $Y$ :

$$
Y=(x-y) \partial_{y}-\partial_{t} .
$$

We aim to approximate operator $K$ by finite differences on the uniform grid

$$
G=\left\{\left(j \Delta_{x}, k \Delta_{y}, n \Delta_{t}\right) \mid j, k, n \in \mathbb{Z}\right\} .
$$

Since $K$ is strongly degenerate, it is known that the classical parabolic boundaryvalue problem for (3.3) is not well posed. In order to comply with the non-Euclidean geometry naturally associated to $K$ (see, for example, Manfredini 1997), we impose

$$
\Delta_{y}=\Delta_{x} \Delta_{t}
$$

Since equation (3.3) is not homogeneous (in the sense of (2.15)), the discrete operator that one might naively associate to $K$ is not well defined on $G$ because it involves points not belonging to the grid. However, it is possible to approximate $K$ efficiently by the following 'corrected' operator:

$$
\begin{aligned}
K_{G} u(x, y, t)=D_{h}^{2} u(x, y+ & \delta(x-h[y / h]), t-\delta) \\
& -\frac{u(x, y, t)-u(x, y+\delta(x-h[y / h]), t-\delta)}{\delta},
\end{aligned}
$$

where $\delta, h>0,[x]$ denotes the integer part of $x \in \mathbb{R}$ and

$$
D_{h}^{2} u(x, y, t)=\frac{u(x+h, y, t)-2 u(x, y, t)+u(x-h, y, t)}{h^{2}}
$$

is the usual second-order central difference. Indeed we have the following lemma.

Proc. R. Soc. Lond. A (2004) 
Lemma 3.1. Operator $K_{G}$ is well defined on the grid $G$ with $\Delta_{x}=h, \Delta_{t}=\delta$ and approximates $K$ in the sense that, for every smooth function $u$, it holds that

$$
\begin{aligned}
\left\|K_{G} u-K u\right\|_{L^{\infty}} \leqslant \Delta_{x}\left\|\partial_{y} u\right\|_{L^{\infty}}+\Delta_{t}\left(\left\|Y^{2} u\right\|_{L^{\infty}}+\left\|Y \partial_{x}^{2} u\right\|_{L^{\infty}}\right) & \\
& +\Delta_{x}^{2}\left\|\partial_{x}^{4} u\right\|_{L^{\infty}}+\Delta_{x} \Delta_{t}\left\|\partial_{x x y} u\right\|_{L^{\infty}} .
\end{aligned}
$$

Proof. By (3.5), the first assertion is obvious. To prove (3.7), we first observe that

$$
0 \leqslant y-h\left[\frac{y}{h}\right]<h, \quad h>0, y \in \mathbb{R} .
$$

Then we have

$$
\begin{aligned}
& \left|\frac{u(x, y+\delta(x-h[y / h]), t-\delta)-u(x, y, t)}{\delta}-Y u(x, y, t)\right| \\
& \leqslant\left|\frac{u(x, y+\delta(x-h[y / h]), t-\delta)-u(x, y+\delta(x-y), t-\delta)}{\delta}\right| \\
& \quad+\left|\frac{u(x, y+\delta(x-y), t-\delta)-u(x, y, t)}{\delta}-Y u(x, y, t)\right|
\end{aligned}
$$

(by the mean value theorem and (3.8))

$$
\leqslant h\left\|\partial_{y} u\right\|_{L^{\infty}}+\delta\left\|Y^{2} u\right\|_{L^{\infty}} .
$$

An analogous estimate for the second-order part of $K$ completes the proof.

Lemma 3.2 (maximum principle). Let $v$ be a solution to the discrete Cauchy problem

$$
\begin{aligned}
K_{G} v=f & \text { in } S_{T} \cap G, \\
v(\cdot, 0)=\psi & \text { in } G_{0},
\end{aligned}
$$

where $f, \psi$ are bounded functions and $G_{0}=G=\left\{\left(j \Delta_{x}, k \Delta_{y}\right) \mid j, k \in \mathbb{Z}\right\}$. If the stability condition

$$
\frac{\Delta_{t}}{\left(\Delta_{x}\right)^{2}} \leqslant \frac{1}{2}
$$

holds, then

$$
\sup _{G \cap S_{T}}|v| \leqslant \sup _{G_{0}}|\psi|+T \sup _{G \cap S_{T}}|f| .
$$

Proof. Let us denote

$$
v_{j, k}^{n}=v\left(j \Delta_{x}, k \Delta_{y}, n \Delta_{t}\right), \quad j, k, n \in \mathbb{Z} .
$$

Once we have observed that (3.10) is equivalent to

$$
\begin{aligned}
v_{j, k}^{n+1}=\left(1-2 \frac{\Delta_{t}}{\left(\Delta_{x}\right)^{2}}\right) & v_{j, k+j-\left[k \Delta_{t}\right]}^{n} \\
& +\frac{\Delta_{t}}{\left(\Delta_{x}\right)^{2}}\left(v_{j, k+j-\left[k \Delta_{t}\right]}^{n}+v_{j, k-j-\left[k \Delta_{t}\right]}^{n}\right)-\Delta_{t} f_{j, k+j-\left[k \Delta_{t}\right]}^{n},
\end{aligned}
$$

for $j, k, n \in \mathbb{Z}$ with $0 \leqslant n \Delta_{t}<T$, the thesis follows by a standard induction argument. 
Lemma 3.3. If $\varphi$ is Lipschitz continuous in $\mathbb{R}^{N}$ and $u$ is a solution to problem (2.18), then for every $s \in] 0, T[$ we have

$$
|u(x, t+s)-u(x, t)| \leqslant c(1+|x|) \sqrt{s}, \quad \forall(x, t) \in S_{T-s},
$$

where the constant $c$ only depends on $K, T$ and the Lipschitz constant of $\varphi$.

Proof. By the representation formula (2.17) it suffices to consider the case $t=0$. Then, if $C=C(t)$ denotes the matrix in (2.13) with $A=\mu I_{p}$, by $(2.17)$ and $(2.19)$ we infer that

$$
\begin{aligned}
|u(x, s)-\varphi(x)| & \leqslant \frac{c}{\sqrt{\operatorname{det} C(s)}} \int_{\mathbb{R}^{N}} \mathrm{e}^{-\left\langle C^{-1}(s)(x-E(s) y), x-E(s) y\right\rangle / 4}|\varphi(y)-\varphi(x)| \mathrm{d} y \\
& =\bar{c} \int_{\mathbb{R}^{N}} \mathrm{e}^{-|\xi|^{2} / 4}\left|\varphi\left(E(-s)\left(x-C^{1 / 2}(s) \xi\right)\right)-\varphi(x)\right| \mathrm{d} \xi
\end{aligned}
$$

(by the change of variables $\xi=C^{-1 / 2}(s)(x-E(s) y)$ ) and the thesis follows by the Lipschitz continuity of $\varphi$.

We are now in a position to prove the following convergence result.

Theorem 3.4. Let $u$ be a solution to the Cauchy problem (3.3), (3.2) with initial datum $\varphi$ bounded and Lipschitz continuous. Let $u_{G}$ be a solution to the correspondent discrete problem (3.10), (3.11) with $f=0$ and $\psi=\varphi$. Assume the stability condition (3.12). Then $u_{G}$ tends to $u$ uniformly on compacts.

Proof. For a fixed suitably small $s>0$, we define

$$
u^{s}(x, t)=u(x, t+s), \quad(x, t) \in S_{T-s}
$$

and we denote by $u_{G}^{s}$ the solution to $(3.10),(3.11)$ with $f=0$ and $\psi=u^{s}(\cdot, 0)$. Let $M$ be a compact subset of $\left.\mathbb{R}^{2} \times\right] 0, T[$. By lemma 3.3 , we have

$$
\sup _{z \in M}\left|u(z)-u^{s}(z)\right| \leqslant c \sqrt{s}
$$

Moreover, keeping in mind that $\partial_{y}=\left[\partial_{x}, Y\right]$ is a third-order derivative, by the representation formula (2.17) and estimate (2.19), we have

$$
\left.\begin{array}{r}
\sup _{S_{T-s}}\left|\partial_{y} u^{s}\right| \leqslant c s^{-3 / 2} \sup |\varphi|, \\
\sup _{S_{T-s}}\left(\left|\partial_{x}^{4} u^{s}\right|+\left|Y \partial_{x x} u^{s}\right|+\left|Y^{2} u^{s}\right|\right) \leqslant c s^{-2} \sup |\varphi|, \\
\sup _{S_{T-s}}\left|\partial_{x x y} u^{s}\right| \leqslant c s^{-5 / 2} \sup |\varphi|,
\end{array}\right\}
$$

with $c$ independent of $s$. Now, the function $v=u^{s}-u_{G}^{s}$ is a solution to (3.10), (3.11) with $f=K_{G} u^{s}-K u^{s}$ and initial datum $\psi=0$. Therefore, applying to $v$ the maximum principle and lemma 3.1 combined with the estimates (3.16) and the stability condition (3.12), we get

$$
\sup _{G \cap S_{T-s}}\left|u^{s}-u_{G}^{s}\right| \leqslant c\left(\Delta_{x} s^{-3 / 2}+\Delta_{x}^{2} s^{-2}+\Delta_{x}^{3} s^{-5 / 2}\right),
$$

with $c=c(\varphi)$.

Proc. R. Soc. Lond. A (2004) 
Finally, $u_{G}^{s}-u_{G}$ is a solution to (3.10), (3.11) with $f=0$ and initial datum $\psi=u(\cdot, s)-\varphi$. By $(3.15),|\psi| \leqslant c s^{1 / 2}$. Hence, by the maximum principle, we have

$$
\sup _{G \cap S_{T-s}}\left|u_{G}^{s}-u_{G}\right| \leqslant c \sqrt{s} .
$$

Combining (3.15), (3.17) and (3.18), we deduce

$$
\sup _{G \cap M}\left|u-u_{G}\right| \leqslant c\left(\sqrt{s}+\Delta_{x} s^{-3 / 2}+\Delta_{x}^{2} s^{-2}+\Delta_{x}^{3} s^{-5 / 2}\right),
$$

and, by choosing $s=\sqrt{\Delta_{x}}$, we get

$$
\max _{M \cap G}\left|u-u_{G}\right| \leqslant c\left(\Delta_{x}\right)^{1 / 4}
$$

where the positive constant $c$ only depends on $K, M$ and $\varphi$.

Remark 3.5. The rate of convergence of the scheme is given explicitly in (3.19). Clearly, it is not comparable with the classical results for parabolic PDEs and it is consequent to the correction made in the definition of $K_{G}$ in (3.6). As a refinement of the above scheme, one can use linear interpolation and approximate $K$ by the discrete operator

$$
\theta K_{G}+(1-\theta) \tilde{K}_{G}, \quad \theta=\theta(x, y, t) \in[0,1],
$$

where $\tilde{K}_{G}$ is formally defined as in $(3.6)$ with the term $[y / h]$ replaced by $[y / h]+1$. The above technique can also be adapted straightforwardly to implicit difference schemes.

Theorem 3.4 can be generalized straightforwardly to problem (3.1), (3.2). In the definition of the grid, (3.5) should be replaced by $\Delta_{y}=|\alpha| \Delta_{x} \Delta_{t}$, and the approximating operator becomes

$$
\begin{gathered}
K_{G} u(x, y, t)=a(x, y, t) D_{h}^{2} u(x, \tilde{y}, t-\delta) \\
+b(x, y, t) \frac{u(x+h \operatorname{sgn}(b(x, y, t)), \tilde{y}, t-\delta)-u(x, \tilde{y}, t-\delta)}{h \operatorname{sgn}(b(x, y, t))} \\
\quad-\frac{u(x, y, t)-u(x, \tilde{y}, t-\delta)}{\delta},
\end{gathered}
$$

where

$$
\tilde{y}=y+\delta\left(\alpha x+|\alpha| h\left[\frac{\beta y}{|a| h}\right]\right) .
$$

In (3.20), the first-order term $b u_{x}$ is approximated by the usual upwind difference, that is, by using the forward difference where $b$ is positive and the backward difference where it is negative. We then have the following theorem.

Theorem 3.6. Let $u$ be a solution to (3.1), (3.2) and $u_{G}$ a solution to (3.10), (3.11) with $f=0$ and $\psi=\varphi$. Under the stability condition

$$
\frac{\Delta_{t}}{\left(\Delta_{x}\right)^{2}} \leqslant \frac{1}{2 \sup a+\sup |b|}
$$

for every compact subset $M$ of $\left.\mathbb{R}^{2} \times\right] 0, T[$ there exists a positive constant $c$ such that

$$
\sup _{M \cap G}\left|u-u_{G}\right| \leqslant c\left(\Delta_{x}\right)^{1 / 4} \text {. }
$$

This research was supported by the University of Bologna. Funds for selected research topics.

Proc. R. Soc. Lond. A (2004) 


\section{References}

Antonelli, F. \& Pascucci, A. 2002 On the viscosity solutions of a stochastic differential utility problem. J. Diff. Eqns 186, 69-87.

Barucci, E., Polidoro, S. \& Vespri, V. 2001 Some results on partial differential equations and Asian options. Math. Models Meth. Appl. Sci. 11, 475-497.

Barucci, E., Malliavin, P., Mancino, M. E., Renò, R. \& Thalmaier, A. 2003 The price-volatility feedback rate: an implementable mathematical indicator of market stability. Math. Finance 13, $17-36$.

Black, F. \& Scholes, M. 1973 The pricing of options and corporate liabilities. J. Polit. Econ. 81, $637-654$.

Bollerslev, T., Chou, R. Y. \& Kroner, K. F. 1992 ARCH modeling in finance: a review of the theory and empirical evidence. J. Econometrics 52, 5-59.

Cox, J. \& Ross, S. 1976 The valuation of options for alternative stochastic processes. J. Financ. Econ. 3, 145-166.

Davis, M. H. A. 2004 Complete-market models of stochastic volatility. Proc. R. Soc. Lond. A 460, 11-26.

Derman, E. \& Kani, I. 1998 Stochastic implied trees: arbitrage pricing with stochastic term and strike structure of volatility. Int. J. Theor. Appl. Finance 1, 61-110.

Di Francesco, M. \& Pascucci, A. 2004 On a class of degenerate parabolic equations of Kolmogorov type. (Preprint.) (Available at http://amsacta.cib.unibo.it/archive/00000829/.)

Dumas, B., Fleming, J. \& Whaley, R. E. 1998 Implied volatility functions: empirical tests. J. Finance 53, 2059-2106.

Dupire, B. 1997 Pricing and hedging with smiles. In Mathematics of derivative securities (ed. M. A. H. Dempster \& S. R. Pliska), ch. 8, pp. 103-111. Cambridge University Press.

Epps, T. W. 2000 Pricing derivative securities. World Scientific.

Figà-Talamanca, G. \& Guerra, M. L. 2000 Complete models with stochastic volatility: further implications. Working Paper 5, Facoltà di Economia, Università degli Studi della Tuscia, Viterbo, Italy.

Folland, G. B. 1975 Subelliptic estimates and function spaces on nilpotent Lie groups. Ark. Mat. 13, 161-207.

Ghysels, E., Harvey, A. C. \& Renault, E. 1996 Stochastic volatility. In Statistical methods in finance. Handbook of Statistics, vol. 14, pp. 119-191. Amsterdam: North-Holland.

Hobson, D. G. \& Rogers, L. C. G. 1998 Complete models with stochastic volatility. Math. Finance 8, 27-48.

Hörmander, L. 1967 Hypoelliptic second order differential equations. Acta Math. 119, 147-171.

Kolmogorov, A. 1934 Zufällige Bewegungen. (Zur Theorie der Brownschen Bewegung.) Ann. Math. 35, 116-117.

Lanconelli, E. \& Polidoro, S. 1994 On a class of hypoelliptic evolution operators. Rend. Sem. Mat. Univ. Polit. Torino 52, 29-63.

Lanconelli, E., Pascucci, A. \& Polidoro, S. 2002 Linear and nonlinear ultraparabolic equations of Kolmogorov type arising in diffusion theory and in finance. In Nonlinear problems in mathematical physics and related topics, II. International Mathematical Series, vol. 2, pp. 243265. Kluwer.

Manfredini, M. 1997 The Dirichlet problem for a class of ultraparabolic equations. Adv. Diff. Eqns 2, 831-866.

Pascucci, A. \& Polidoro, S. 2003 On the Cauchy problem for a nonlinear Kolmogorov equation. SIAM J. Math. Analysis 35, 579-595.

Peszek, R. 1995 PDE models for pricing stocks and options with memory feedback. Appl. Math. Fin. 2, 53-105.

Proc. R. Soc. Lond. A (2004) 
Polidoro, S. 1994 On a class of ultraparabolic operators of Kolmogorov-Fokker-Planck type. Matematiche (Catania) 49, 53-105.

Rubinstein, M. 1994 Implied binomial trees. J. Finance 49, 771-818.

Tompkins, R. 2001 Stock index futures markets: stochastic volatility models and smiles. J. Fut. Mark. 21, 43-78.

Wilmott, P., Howison, S. \& Dewynne, J. 1993 Option pricing. Oxford Financial Press. 